\title{
Symptomatic Central Venous Stenosis in a Hemodialysis Patient Leading to Loss of Arteriovenous Access: A Case Report and Literature Review
}

\author{
Vasishta S. Tatapudia, b Noam Spinowitz ${ }^{c}$ David S. Goldfarba, b \\ a Nephrology Section, New York Harbor Veterans Affairs Medical Center, \\ ${ }^{b}$ Nephrology Division, New York University School of Medicine, and \\ ${ }^{\mathrm{c}}$ American Access Care, New York, N.Y., USA
}

Key Words

Central venous stenosis · Hemodialysis · Arteriovenous access

\begin{abstract}
Central venous stenosis is a well-described sequel to the placement of hemodialysis catheters in the central venous system. The presence of an ipsilateral arteriovenous fistula or graft often leads to severe venous dilatation, arm edema and recurrent infections. Vascular access thrombosis, compromised blood flow and inadequate dialysis delivery are dreaded complications that eventually render the access unusable. We report the case of a 58-year-old male hemodialysis patient who developed symptomatic central venous stenosis to illustrate the problem and review the pertinent literature. This patient developed severe enlargement of upper extremity veins due to central venous stenosis. The symptoms were refractory to multiple endovascular interventions and eventually necessitated ligation of his arteriovenous fistula. Central venous stenosis remains a pervasive problem despite advances in our understanding of its etiology and recognition of the enormity of its consequences. Due to the lack of effective therapeutic options, prevention is better than cure.

(C) 2014 S. Karger AG, Basel
\end{abstract}

\section{Introduction}

A functioning permanent vascular access that can be regularly utilized to access the systemic circulation is crucial for the survival of patients on maintenance hemodialysis. Yet, hemodialysis access failure was reported to be the most frequent cause of hospitalization among patients with chronic kidney disease stage 5 by the United States Renal Data System. Qualities of an ideal access include the ability to deliver a blood flow rate adequate for the 
Tatapudi et al.: Symptomatic Central Venous Stenosis in a Hemodialysis Patient Leading to Loss of Arteriovenous Access: A Case Report and Literature Review

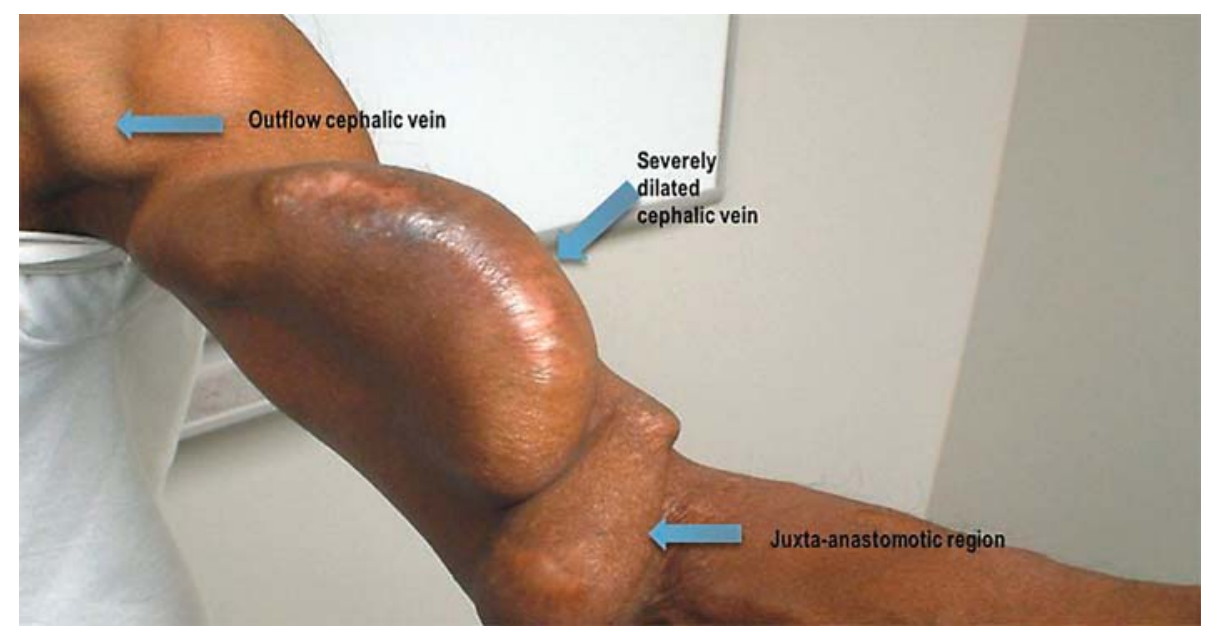

Fig. 1. Massive enlargement of the venous system of the left upper arm.

dialysis prescription, good long-term patency and a low rate of complications such as infection, stenosis, thrombosis, aneurysm and limb ischemia [1]. Studies have shown that arteriovenous fistula survival is superior to grafts when used as patients' first access [2]. Hemodialysis catheters play an important role in the delivery of dialysis to patients with end-stage renal disease. However, anatomic, thrombotic and infectious complications associated with their continued use lead to significant morbidity in this population [3]. Clinical practice guidelines emphasizing the importance of placing fistulae in long-term hemodialysis patients for vascular access were first published by the NKF KDOQI (National Kidney Foundation Kidney Disease Outcomes Quality Initiative) in 1997 [1]. Central venous stenosis related to cannulation of central veins remains common in dialysis patients and is fraught with several complications that may lead to loss of arteriovenous access $[4,5]$.

\section{Case Presentation}

We report the case of a 58-year-old male African-American patient who became dialysis dependent in 1990 after he had developed end-stage renal disease due to focal segmental glomerulosclerosis. He had received hemodialysis via a left-radial-artery-to-brachial-vein Gore-Tex graft for several years before he was noted to have poor arteriovenous access blood flow. Venography showed graft stenosis and an accompanying thrombus. Despite efforts to restore patency, the graft could not be salvaged. A tunneled left subclavian hemodialysis catheter was placed in order to continue maintenance hemodialysis. He subsequently underwent creation of a left brachiocephalic arteriovenous fistula that provided reliable vascular access for hemodialysis during several months. The patient eventually underwent a successful cadaveric kidney transplant and was emancipated from hemodialysis. Months later, he developed a remarkable enlargement of the arteriovenous fistula (fig. 1) and dilated jugular veins (fig. 2). Venography revealed $50 \%$ stenosis of the left brachiocephalic vein accompanied by dilatation of the ipsilateral cephalic and subclavian veins (fig. 3). Retrograde flow was noted in the left internal jugular vein. After successful dilatation of the left brachiocephalic vein stenosis by balloon angioplasty, venography showed restoration of the blood flow in the central venous circulation and disappearance of the previously noted retrograde flow in the left internal jugular vein (fig. 4). However, as the access arm remained massively 
Fig. 2. Engorgement of the jugular veins seen on the left side of the neck.

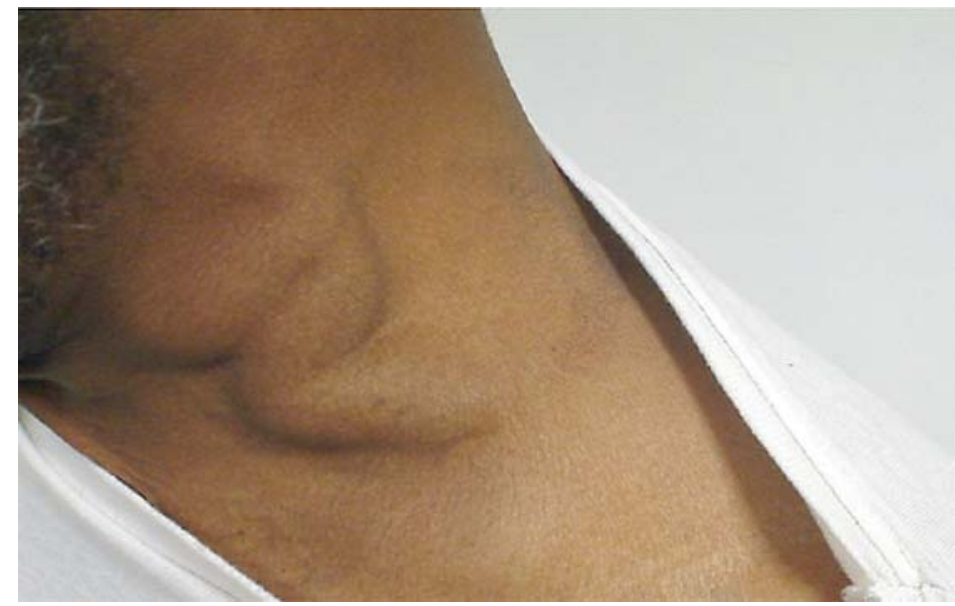

enlarged, venography was repeated a few weeks later, revealing moderate-to-severe stenosis of the left cephalic as well as subclavian veins. Successful balloon angioplasties of both lesions were performed. Despite several interventions, the striking enlargement of the venous system persisted, and surgical ligation of the fistula was done to avert catastrophic complications.

\section{Discussion}

Central venous stenosis is a major, frequently encountered problem in the dialysis population. In a study of 69 consecutive patients being subjected to percutaneous placement of tunneled right internal jugular vein catheters who underwent venography prior to insertion of a guide wire, 29 patients (42\%) were found to have unexpected stenosis or angulation of central veins severe enough to necessitate additional fluoroscopy or abandonment of the procedure [5].

Much of the early attention was focused on hemodialysis catheters as the etiologic agents responsible for this problem [6]. However, insertion of a variety of indwelling devices including central venous catheters, peripherally inserted central venous catheters and indwelling intracardiac wires leads to the development of central venous stenosis [7-9]. Central venous stenosis has also been reported to occur in the absence of any of these antecedents [10]. It has long been recognized that dialysis catheters are associated with late obstructive complications that may adversely affect the outcomes of permanent vascular access. Early case reports in the 1980s linked subclavian dialysis catheters to central venous stenosis [6]. In a prospective study of 50 patients with subclavian catheters and 50 patients with internal jugular vein catheters, venography revealed central venous stenosis in $42 \%$ of the patients in the subclavian group compared with $10 \%$ of the patients in the internal jugular group [11]. However, more recent studies show that, despite embracing preferential cannulation of the internal jugular vein, central venous stenosis remains widespread. In a study of 133 hemodialysis patients who underwent venography for access-related concerns over a 14-month period, 55 patients (41\%) had evidence of significant central venous stenosis. Longer time on hemodialysis (43 vs. 34 months) and a history of a previous hemodialysis catheter insertion (52/55 vs. 59/78 patients) were the factors found to be associated with stenosis [4].

In addition to the site of insertion, the incidence of central venous stenosis is contingent upon the laterality of the insertion and the type of the indwelling object, i.e. a central venous catheter, peripherally inserted central venous catheter or intracardiac wire. A tendency toward 


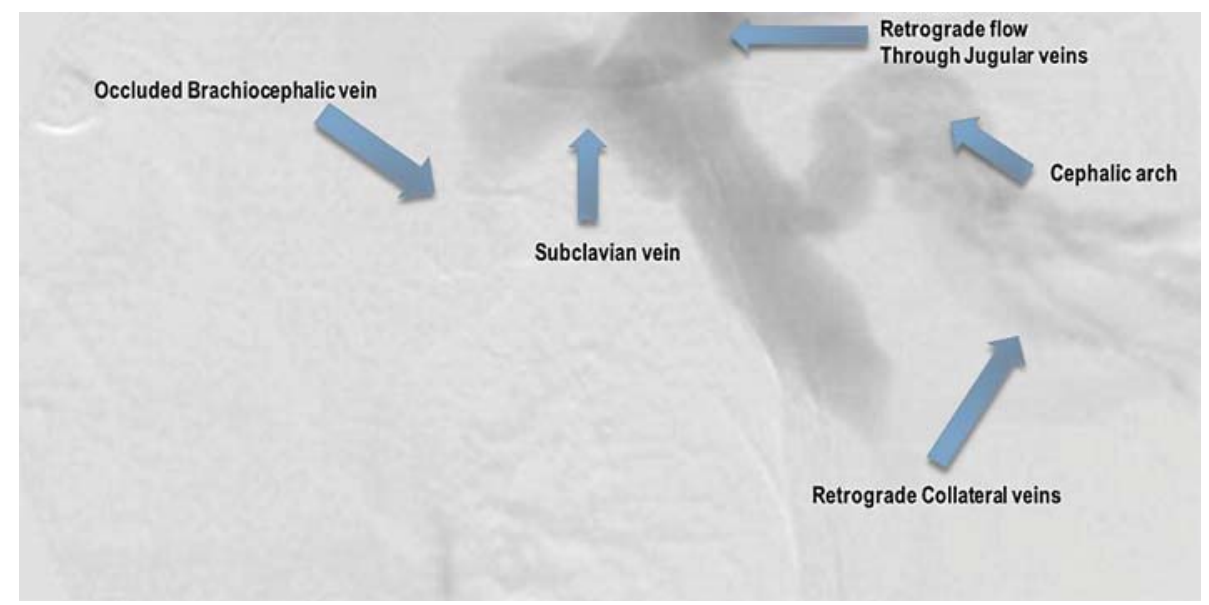

Fig. 3. Venogram showing aneurysmal dilatation of the cephalic arch and subclavian vein with complete occlusion of the brachiocephalic vein.

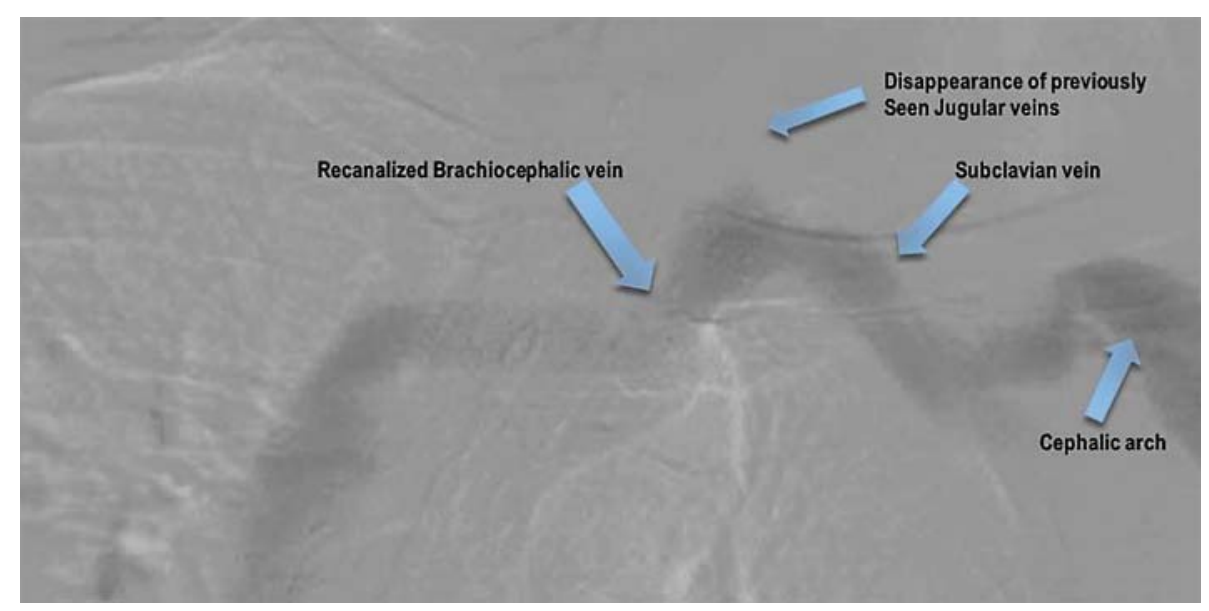

Fig. 4. Venogram after angioplasty showing improved venous outflow to the central circulation. Note the disappearance of the previously retrograde collateral veins.

an increased incidence of stenosis was observed in hemodialysis patients following cannulation of the left compared with the right internal jugular vein $[11,12]$. This led to the conjecture that the angulations between the left internal jugular vein, brachiocephalic vein and superior vena cava that the catheter must negotiate, as well as the greater length of the path traversed by a left-sided when compared with a right-sided catheter, are responsible for this increased incidence $[12,13]$. The complex three-dimensional anatomy of the left central veins has also been implicated in the increased incidence of stenosis related to left-sided central venous catheters. Angulation of the left brachiocephalic vein as it winds around the aortic arch is not apparent on two-dimensional radiographs or venograms. Contact between the catheter and vessel wall as it traverses this fulcrum is thought to lead to endothelial irritation and possible subsequent venous stenosis [13]. Therefore, the KDOQI guidelines opine that the right internal jugular vein is the preferred site for the insertion of tunneled cuffed venous dialysis catheters [1].

A majority of the affected patients remain asymptomatic. In a study of 202 patients who underwent permanent pacemaker implantations with indwelling intracardiac wires inserted 
Tatapudi et al.: Symptomatic Central Venous Stenosis in a Hemodialysis Patient

Leading to Loss of Arteriovenous Access: A Case Report and Literature Review

via a transvenous approach, 129 patients (64\%) developed varying degrees of central venous stenosis. However, symptoms were present in only 12 patients (9.3\%) [8]. Central venous stenosis becomes clinically significant in the presence of an ipsilateral arteriovenous dialysis access that drains into the affected central veins. The high venous pressure and blood flow due to the fistula may overwhelm the collateral venous and lymphatic drainage, resulting in the development of dilated and tortuous collateral veins over the ipsilateral upper arm, neck and upper chest [6]. In severe cases, venous hypertension may eventually lead to disabling arm edema with pain and discomfort.

Vascular access stenosis is a harbinger of thrombosis, recurrent infections, reduced access blood flow and compromised dialysis delivery due to access recirculation [14]. Myriad complications can render the arteriovenous access unusable despite valiant efforts to salvage the access by endovascular and surgical therapies, leaving no choice but to occlude the access. A great majority of patients who face this predicament may not be as fortunate as our patient, who received a kidney transplant. KDOQI guidelines steadfastly recommend that fistula placement should be considered first, followed by prosthetic grafts if fistula placement is not possible in prospective hemodialysis patients. These guidelines also discourage the use of catheters unless other options are unavailable [1]. Indeed, some authors recommend a 'catheter-last' approach after having exhausted all avenues including fistula, graft, peritoneal dialysis and preemptive kidney transplantation [15]. Hence, every effort must be made to prevent central venous stenosis.

\section{References}

1 National Kidney Foundation KDOQI: 2006 updates: clinical practice guidelines and recommendations. http:// www.kidney.org/professionals/kdoqi/pdf/12-50-0210_JAG_DCP_Guidelines-VA_Oct06_SectionC_ofC.pdf (accessed August 29, 2013).

-2 Pisoni RI, Young EW, Dykstra DM, et al: Vascular access use in Europe and the United States: results from the DOPPS. Kidney Int 2002;61:305-316.

3 Schwab SJ, Beathard G: The hemodialysis catheter conundrum: hate living with them, but can't live without them. Kidney Int 1999;56:1-17.

4 Macrae JM, Ahmed A, Johnson N, Levin A, Kiaii M: Central vein stenosis: a common problem in patients on hemodialysis. ASAIO J 2005;51:77-81.

5 Taal MW, Chesterton LJ, McIntyre CW: Venography at insertion of tunnelled internal jugular vein dialysis catheters reveals significant occult stenosis. Nephrol Dial Transplant 2004;19:1542-1545.

6 Davis D, Petersen J, Feldman R, Cho C, Stevick CA: Subclavian venous stenosis: a complication of subclavian dialysis. JAMA 1984;252:3404-3406.

7 Gonsalves CF, Eschelman DJ, Sullivan KL, DuBois N, Bonn J: Incidence of central vein stenosis and occlusion following upper extremity PICC and port placement. Cardiovasc Intervent Radiol 2003;26:123-127.

-8 da Costa SS, Scalabrini Neto A, Costa R, Caldas JG, Martinelli Filho M: Incidence and risk factors of upper extremity deep vein lesions after permanent transvenous pacemaker implant: a 6-month follow-up prospective study. Pacing Clin Electrophysiol 2002;25:1301-1306.

$>9$ Teruya TH, Abou-Zamzam AM Jr, Limm W, Wong L, Wong L: Symptomatic subclavian vein stenosis and occlusion in hemodialysis patients with transvenous pacemakers. Ann Vasc Surg 2003;17:526-529.

10 Oguzkurt L, Tercan F, Yıldırım S, Torun D: Central venous stenosis in haemodialysis patients without a previous history of catheter placement. Eur J Radiol 2005;55:237-242.

11 Schillinger F, Schillinger D, Montagnac R, Milcent T: Post catheterisation vein stenosis in haemodialysis: comparative angiographic study of 50 subclavian and 50 internal jugular accesses. Nephrol Dial Transplant 1991;6:722-724.

12 Salgado OJ, Urdaneta B, Colmenares B, García R, Flores C: Right versus left internal jugular vein catheterization for hemodialysis: complications and impact on ipsilateral access creation. Artif Organs 2004;28:728-733.

13 Salik E, Daftary A, Tal MG: Three-dimensional anatomy of the left central veins: implications for dialysis catheter placement. J Vasc Interv Radiol 2007;18:361-364.

14 Kumar V, Depner T, Besarab A, Ananthakrishnan S: Arteriovenous access for hemodialysis; in Daurgidas JT, Blake PG, Ing TS (eds): Handbook of Dialysis, ed 4. Philadelphia, Lippincott Williams \& Wilkins, 2007, pp 105-126.

15 Agarwal AK: Central vein stenosis. Am J Kidney Dis 2013;61:1001-1015. 